\title{
Immunohistochemical evaluation of the heat shock response to nonablative fractional resurfacing
}

Basil M. Hantash

Elixir Institute of Regenerative Medicine

5941 Optical Court

San Jose, California 95138

Vikramaditya P. Bedi

Solta Medical, Incorporated

25881 Industrial Boulevard

Hayward, California 94545

Steven K. Struck

Struck Plastic Surgery

331 El Camino Real, Suite 200

Atherton, California 94027

Kin F. Chan

Solta Medical, Incorporated 25881 Industrial Boulevard Hayward, California 94545

\begin{abstract}
Despite the emergence of nonablative fractional resurfacing (NFR) as a new therapeutic modality for skin photoaging, little is known about the molecular events that underlie the heat shock response to different treatment parameters. Human subjects are treated with a scanned 1550-nm fractional laser at pulse energies spanning 6 to $40 \mathrm{~mJ}$ and a $140-\mu \mathrm{m}$ spot size. The heat shock response is assessed immunohistochemically immediately through 7 days posttreatment. At the immediately posttreatment time point, we observe subepidermal clefting in most sections. The basal epidermis and dermal zones of sparing are both found to express HSP47, but not HSP72. By day 1, expression of HSP72 is detected throughout the epidermis, while that of HSP47 remains restricted to the basal layer. Both proteins are detected surrounding the dermal portion of the microscopic treatment zone (MTZ). This pattern of expression persists through day 7 post-NFR, although neither protein is found within the MTZ. Immediately posttreatment, the mean collagen denaturation zone width is $50 \mu \mathrm{m}$ at $6 \mathrm{~mJ}$, increasing to $202 \mu \mathrm{m}$ at $40 \mathrm{~mJ}$. The zone of cell death exceeds the denaturation zone by 19 to $55 \%$ over this pulse energy range. The two zones converge by day 7 posttreatment. @ 2010 The International Society for Optical Engineering. [DOI: 10.1117/1.3526355]
\end{abstract}

Keywords: collagen; fractional resurfacing; heat shock protein; nonablative.

Paper 10361R received Jun. 28, 2010; revised manuscript received Sep. 18, 2010; accepted for publication Oct. 28, 2010; published online Dec. 20, 2010.

\section{Introduction}

Lasers have now safely been employed for the treatment of a variety of skin conditions for several decades. With an aging baby boomer population, physicians have witnessed increasing demand for more aesthetic-related procedures. Until recently, laser devices relied exclusively on the principle of selective photothermolysis to achieve their clinical outcomes. ${ }^{1}$ Although this modality has been used for the treatment of rhytides, melasma, photodamage, and scars, efficacy has been quite variable and often unpredictable. ${ }^{2}$ In addition, adverse events such as hyperpigmentation often have limited use in patients with darker skin types.

In 2004, a novel nonablative laser device utilizing the principle of fractional photothermolysis (FP) was introduced. ${ }^{3}$ This laser utilizes an erbium-glass fiber laser emitting at $1550 \mathrm{~nm}$ that is coupled with a scanning handpiece. By treating only a fraction of the total surface area of skin, the laser achieves a reduction in side effects such as postinflammatory hyperpigmentation, enabling its use across all skin types. FP has demonstrated clinical efficacy for a wide array of clinical indications and was recently reviewed. ${ }^{4}$ In particular, several reports suggest a significant benefit in improving the clinical appearance of scars. ${ }^{5-8}$ Indeed, previous studies showed significant improvement in the appearance of post-operative scars after laser assisted skin closure. $^{9-12}$ Using an 815-nm diode laser at subthreshold pulses,

Address all correspondence to: Basil M. Hantash, Elixir Institute of Regenerative Medicine, 5941 Optical Court, San Jose, California 95138. Tel: 408-914-2033. Fax: 408-914-2033; E-mail: basil@elixirinstitute.org. the authors reported upregulation of transforming growth factor beta and subsequent induction of the heat shock protein (HSP) response. Thus, HSPs appear to play an important role in the wound healing response following nonablative laser treatment. In fact, the heat shock response emanating from the interlesional spared tissue is thought to be highly sensitive to temperature rise and was previously shown to result in overexpression of HSP70, which subsequently helps orchestrate the appropriate inflammatory response and wound healing.

Currently, however, very little is known about the heat shock response postnonablative fractional resurfacing (NFR). Since the key advantage of fractional laser treatment rests in its ability to avoid bulk heating and the maintenance of viable tissue capable of contributing to the wound healing response, we recently characterized the effect of NFR on microscopic treatment zone (MTZ) dimensions both ex and in vivo. ${ }^{13}$ Our study revealed that the zone of cell death (ZCD) always exceeded the collagen denaturation zone (CDZ) at all treatment parameters explored. Based on that work, we were able to develop a dosimetry chart that correlated the percentage of surface area coverage to pulse energy. These data also guided design of preselected treatment parameters available to laser users, helping simplify the treatment approach. Although insightful, it remains unclear whether the $\mathrm{CDZ}$ or ZCD is the best-suited biomarker to guide laser operators in the choice of treatment parameters, especially since previous studies suggest that heat shock zones may comprise a much larger volume of affected tissue than that measured for either of the currently used criteria. Moreover, utilizing the

$1083-3668 / 2010 / 15(6) / 068002 / 6 / \$ 25.00$ 
CDZ may be an appropriate predictor of desired clinical endpoints such as collagen remodeling, but offers no direct connection to the risk of bulk heating. Similarly, utilizing the ZCD may offer insight regarding the risk of bulk heating, but no direct correlation with clinical efficacy. Thus, the choice of biomarker (denatured collagen, cell death, or HSP expression) can dramatically alter the treatment parameter optimization process by affecting the design of preselected treatment settings and guidelines on the timing of follow-up treatments.

To better understand the precise spatiotemporal nature of the heat shock response post-NFR and its correlation with historical biomarkers such as denatured collagen or cell death, we studied the HSP expression pattern in human subjects up to 7 days following treatment with NFR. The HSP results were compared with measurements of CDZs and ZCDs, and the implications of our findings for epidermal and dermal remodeling as well as potential biomarkers for formulating more useful dosimetry guidelines are discussed.

\section{Methods}

Four healthy subjects of Fitzpatrick skin types II to IV received multiple treatments from a 1550 -nm Fraxel ${ }^{\circledR}$ SR laser system (Solta Medical, Inc., Hayward, California) immediately and 1, 3 , and 7 day(s) prior to abdominoplasty. The study protocol was approved by an Institutional Review Board, and each subject consented prior to participation in the study. Hairs within the designated test areas $\left(\sim 12 \mathrm{~cm}^{2}\right)$ were removed by shaving and either a local anesthetic [for skin excision 1, 3, or 7 day(s) posttreatment] or a general anesthetic was administered (for skin excision immediately posttreatment).

The laser was operated in the standard user mode, allowing for deposition of a constant density of microbeams within a specific range of handpiece velocities. The laser delivered an array of single mode Gaussian microbeams that were $140 \mu \mathrm{m}$ in diameter at the $1 / e^{2}$ incidence beam waist. Pulse energies were varied from 6 to $40 \mathrm{~mJ}$ with multiple passes made by the laser handpiece to produce final spot densities of 250 to $1000 \mathrm{MTZs} / \mathrm{cm}^{2}$. Higher density treatments were used for lower pulse energies and vice versa. The full set of treatment parameters is shown in Table 1.

Immediately following surgical excision, each treatment site was grossed and then either frozen embedded in optimal cutting temperature compound (IMEB Inc., San Marcos, California) or paraffin embedded. The frozen embedded samples were serially sectioned into $12-\mu \mathrm{m}$-thick slices and each adjacent section was stained with hematoxylin and eosin (H\&E) and lactate dehydrogenase (LDH) to measure the $\mathrm{CDZ}$ and $\mathrm{ZCD}$. The paraffin-embedded samples were sectioned into $10-\mu \mathrm{m}$ thick slices and stained with antibodies (Abcam, Cambridge, Massachusetts) to HSP72 or HSP47 at the appropriate dilutions. A total of 50 lesions for each treatment parameter was imaged and recorded using a DM LM/P microscope and a DFC320 digital camera (Leica Microsystem, Cambridge, United Kingdom). Lesion dimension measurements were made with a proprietary Visual Basic ${ }^{\circledR}$ computer program ${ }^{13}$ (Solta Medical, Inc., Hayward, California). Statistical significance was determined using a Student's $t$ test (Microsoft Excel, Microsoft, Seattle, Washington) with $P$ values of less than or equal to 0.05 taken as significant.
Table 1 Treatment parameters.

\begin{tabular}{lll}
\hline Pulse Energy $(\mathrm{mJ})$ & Spot Density $\left(\mathrm{MTZ} / \mathrm{cm}^{2}\right)$ & Number of Passes \\
\hline 6 & 1000 & 1 \\
10 & 1000 & 1 \\
12 & 1000 & 1 \\
15 & 500 & 2 \\
20 & 500 & 2 \\
25 & 500 & 2 \\
30 & 250 & 4 \\
35 & 250 & 4 \\
40 & 250 & 4 \\
\hline
\end{tabular}

\section{Results}

To characterize the heat shock response post-NFR treatment, human subjects were treated at various time periods prior to abdominoplasty, and harvested tissue was examined immunohistochemically for the spatiotemporal HSP expression pattern immediately, and 1, 3, and 7 day(s) post-NFR treatment. Figure 1(a) shows a typical H\&E-stained section from a human
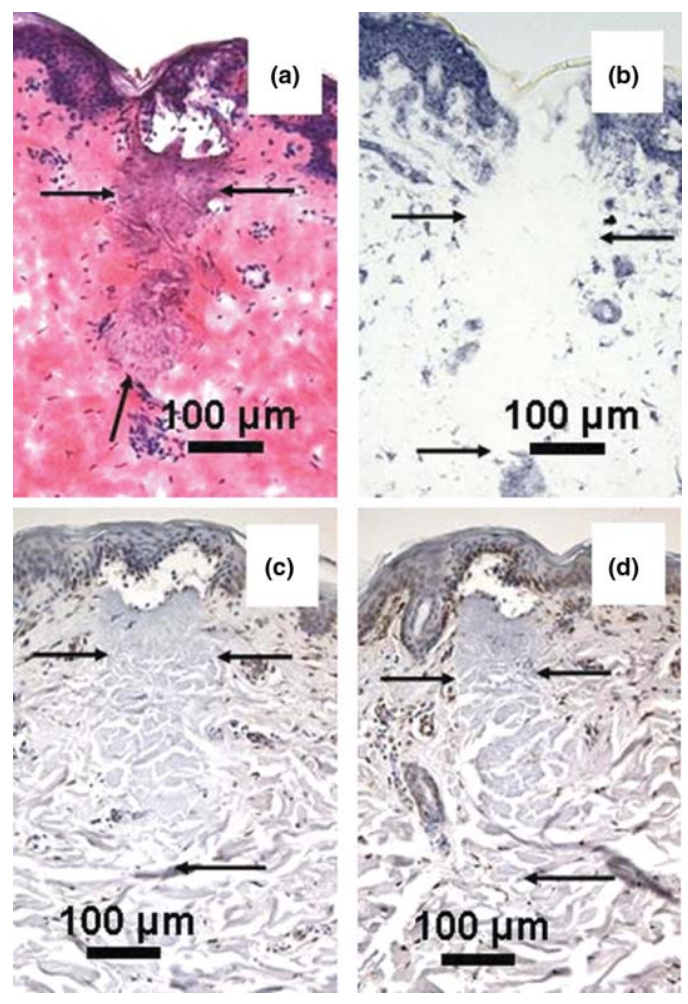

Fig. 1 Immunohistochemistry of human skin immediately post-NFR treatment. In vivo human skin was treated with a 1550-nm infrared fractionated laser then biopsied immediately posttreatment. Tissue was stained with (a) H\&E, (b) LDH, (c) anti-HSP72 antibody, or (d) antiHSP47 antibody. Brown staining in panels (c) and (d) represents positive immunoreactivity. Original magnification x 10 . 
Hantash et al.: Immunohistochemical evaluation of the heat shock response...

Table 2 Lesion width immediately. 1, and 7 day(s) postnonablative treatment.

\begin{tabular}{|c|c|c|c|c|c|c|c|c|c|}
\hline \multirow{3}{*}{$\begin{array}{l}\text { Pulse } \\
\text { Energy } \\
\text { (mJ) }\end{array}$} & \multicolumn{9}{|c|}{ MTZ Width $(\mu \mathrm{m})$} \\
\hline & \multicolumn{3}{|c|}{ Immediate } & \multicolumn{3}{|c|}{ Day 1} & \multicolumn{3}{|c|}{ Day 7} \\
\hline & CDZ & $Z C D$ & $\Delta^{1}$ & $\mathrm{CDZ}$ & $Z C D$ & $\Delta^{2}$ & $\mathrm{CDZ}$ & ZCD & $\Delta^{3}$ \\
\hline 6 & 50 & 63 & $26 \%$ & 39 & 57 & $46 \%$ & 80 & 80 & $0 \%$ \\
\hline 10 & 85 & 122 & $44 \%$ & 80 & 86 & $8 \%$ & $\mathrm{n} / \mathrm{a}$ & $\mathrm{n} / \mathrm{a}$ & $\mathrm{n} / \mathrm{a}$ \\
\hline 12 & 95 & 147 & $55 \%$ & 83 & 101 & $22 \%$ & $\mathrm{n} / \mathrm{a}$ & $n / a$ & $\mathrm{n} / \mathrm{a}$ \\
\hline 15 & 113 & 151 & $34 \%$ & 100 & 120 & $20 \%$ & 135 & 120 & $-11 \%$ \\
\hline 20 & 135 & 188 & $39 \%$ & 126 & 158 & $25 \%$ & 139 & 148 & $6 \%$ \\
\hline 25 & 165 & 220 & $33 \%$ & 137 & 167 & $22 \%$ & $\mathrm{n} / \mathrm{a}$ & $n / a$ & $\mathrm{n} / \mathrm{a}$ \\
\hline 30 & 170 & 225 & $32 \%$ & 143 & 180 & $26 \%$ & 165 & 168 & $2 \%$ \\
\hline 35 & 185 & 239 & $23 \%$ & 172 & 200 & $16 \%$ & $\mathrm{n} / \mathrm{a}$ & $n / a$ & $\mathrm{n} / \mathrm{a}$ \\
\hline 40 & 202 & 250 & $19 \%$ & 160 & 173 & $8 \%$ & 175 & 178 & $2 \%$ \\
\hline
\end{tabular}

${ }^{1} p \leq 0.001,{ }^{2} p \leq 0.05,{ }^{3} p \geq 0.1$

${ }^{2} \mathrm{CDZ}=$ collagen denaturation zone, $\mathrm{MTZ}=$ microscopic treatment zone, $\mathrm{ZCD}=$ zone of cell death, $\Delta=\%$ difference between $C D Z$ and $Z C D, n / a=n o t$ available.

subject immediately post-NFR treatment. No microscopic epidermal necrotic debris (MEND) was present by this time. The border of each MTZ constituting the CDZ could be discerned by the homogenized hypereosinophilic staining dermal material. CDZ width ranged from 50 to $202 \mu \mathrm{m}$ for pulse energies of 6 to $40 \mathrm{~mJ}$ (Fig. 5 and Table 2 at the end of this section). A serial section stained with LDH is shown in Fig. 1(b) and demonstrates the ZCD. The width of the ZCD statistically significantly $(p \leq 0.001)$ exceeded that of the CDZ by as low as $19 \%$ at $40 \mathrm{~mJ}$ to as high as $55 \%$ at $12 \mathrm{~mJ}$ (Fig. 5 and Table 2 at the end of this section).

Under these conditions, treatment with monoclonal antibody to human HSP72 resulted in minimum to no staining in the epidermis [Fig. 1(c)]. In contrast, we detected HSP47 expression in the basal layer of the epidermis [Fig. 1(d)]. Scant HSP72 and HSP47 expression was observed in the dermal zones of sparing. At this juncture, the dermis was notable for minimal cellularity, indicating absence of inflammatory cell recruitment. Consistent with our previous study, ${ }^{13}$ we also observed disruption of the dermal-epidermal (DE) junction, or subepidermal clefting, in most sections examined [(Figs. 1(a), 1(c) and 1(d)].

As shown in Fig. 2(a) and previously reported, ${ }^{13}$ the DE junction was partially restored and the epidermis fully reepithelialized by day 1 posttreatment. A well-formed MEND could be seen just underlying the stratum corneum. By this time, expression of HSP72 was detected throughout the epidermis [Fig. 2(c)], while that of HSP47 remained restricted to the basal layer [Fig. 2(d)]. Both proteins were detected surrounding the dermal portion of the MTZ, but within the CDZ. Dermal cellularity was increased, as evidenced by the basophilic nuclear staining; however, this new infiltrate primarily was found in the spared zones. By day 1, CDZ width was already decreasing and ranged from 39 to $172 \mu \mathrm{m}$ for pulse energies of 6 to $40 \mathrm{~mJ}$ (Fig. 5 and Table 2 at the end of this section). The width of the ZCD exceeded that of the CDZ by a smaller but statistically significant $(p<0.05)$ margin ( 8 to $46 \%)$ than that observed immediately posttreatment.

At 3 days posttreatment, the DE junction was almost completely restored and the MEND was now within the stratum corneum [Fig. 3(a)]. HSP72 expression in the epidermis became more concentrated in the granular layer [Fig. 3(c)], while that of HSP47 remained in the basal epidermis [Fig. 3(d)]. Both HSPs were detected at increased levels in the zone around but not within each MTZ, consistent with the mild increase in cellular density in the dermis.

At 7 days posttreatment, no evidence of subepidermal clefting was observed in any of the sections examined, although the MEND remained attached to the stratum corneum (Fig. 4). HSP72 expression in the epidermis appeared diffuse [Fig. 4(c)], whereas the pattern of HSP47 epidermal expression remained unchanged [Fig. 4(d)]. Both HSPs were detected within the MEND itself for the first time.

The intensity of dermal expression of both HSPs remained unchanged [Figs. 4(c) and 4(d)]. Although cellular density decreased relative to day 3 , HSP72 and 47 staining could now be seen within the CDZ. By day 7, CDZ and ZCD widths were nearly identical $(p>0.1)$ at 80 to 175 and 80 to $178 \mu \mathrm{m}$, respectively, for pulse energies of 6 to $40 \mathrm{~mJ}$ (Fig. 5 and Table 2).

\section{Discussion}

FP represents the latest advance in laser surgery. ${ }^{3}$ Currently, this method has been used successfully to treat numerous dermatological conditions including melasma, poikiloderma, acne scars, and rhytides. ${ }^{4}$ While the mechanism underlying FP's clinical efficacy is uniformly thought to involve dermal collagen coagulation in combination with induction of rapid epidermal 

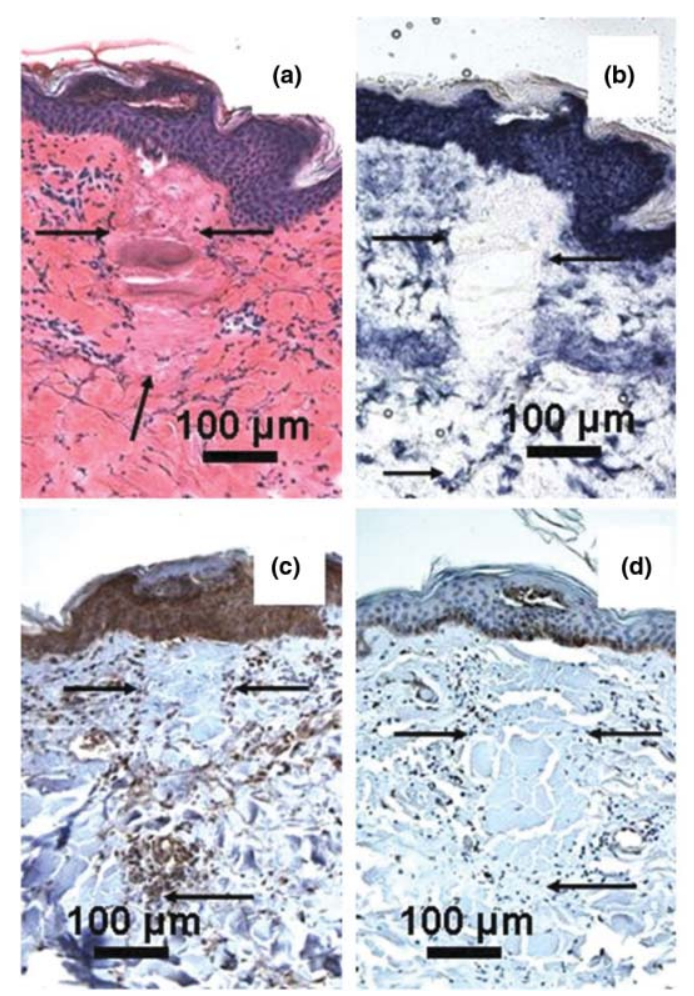

Fig. 2 Immunohistochemistry of human skin 1 day post-NFR treatment. In vivo human skin was treated with a 1550-nm infrared fractionated laser then biopsied immediately posttreatment. Tissue was stained with (a) H\&E, (b) LDH, (c) anti-HSP72 antibody, or (d) anti-HSP47 antibody. Brown staining in panels (c) and (d) represents positive immunoreactivity. Original magnification x 10 .

turnover, the variety of clinical indications just noted often requires unique treatment regimens, each potentially targeting different depths of thermal injury.

In our previous studies, we found that depths of nearly $1 \mathrm{~mm}$ in skin were possible with NFR, in line with the depth of penetration required for effective dermal remodeling. ${ }^{13} \mathrm{We}$ also characterized the relationship between percentage surface area coverage and pulse energy. The former was assessed using two unique biomarkers: denatured collagen and cell death. Our data revealed that the ZCD exceeded the $\mathrm{CDZ}$ at all treatment parameters. Previous studies, however, have suggested that the heat shock response may extend well beyond the treatment zone, leaving open the question of which biomarker is the most suitable choice to guide parameter selection. To help clarify this question, we assessed the spatiotemporal expression pattern of HSPs and compared it to that observed for traditional biomarkers such as the CDZ and ZCD.

Thermal injury to living cells leads to protein coagulation, unfolding, aggregation, and ultimately apoptosis. A critical component of the early heat shock response in skin is the HSP70 family of proteins. ${ }^{14,15}$ HSP70 proteins are thought to protect sublethally damaged cells by stabilizing 3-D protein folding. HSP70 is comprised of HSP72 and 73, both of which are located in the cytoplasm and nucleus of skin cells such as keratinocytes, fibroblasts, and adipocytes. While HSP73 is constitutively expressed by all mammalian cells, HSP72 expression is restricted to situations of stress. We therefore examined the expression pattern of HSP72 post-NFR treatment.
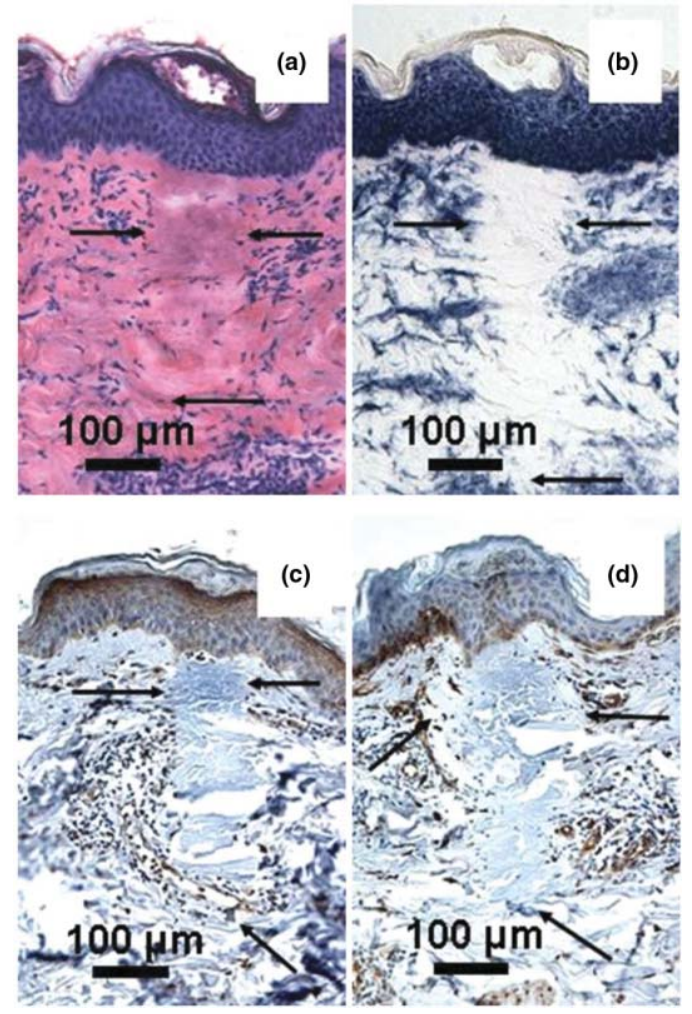

Fig. 3 Immunohistochemistry of human skin 3 days post-NFR treatment. In vivo human skin was treated with a 1550-nm infrared fractionated laser then biopsied immediately posttreatment. Tissue was stained with (a) H\&E, (b) LDH, (c) anti-HSP72 antibody, or (d) anti-HSP47 antibody. Brown staining in panels (c) and (d) represents positive immunoreactivity. Original magnification x 10 .

At baseline and immediately posttreatment, we found little to no expression of HSP72 [Fig. 1(c)]. This is consistent with the findings of Laubach et al., ${ }^{16}$ although this group did not discriminate between different HSP70 family members. By day 1 posttreatment, HSP72 expression was significantly upregulated and found diffusely throughout the epidermis [Fig. 2(c)]. Interestingly, there appeared to be two phases to HSP72 upregulation, one initiated between 0 and $24 \mathrm{~h}$ posttreatment, and a second between days 3 and 7 posttreatment. This was evidenced by the shift from diffuse to focal (granular layer) to diffuse staining at days 1,3 , and 7 respectively.

HSP47 is thought to play a direct role in dermal remodeling, with expression being directly proportional to the rate of neocollagenesis. ${ }^{16-20}$ In line with this, subcellular localization studies revealed that HSP47 expression is restricted to the endoplasmic reticulum of fibroblasts. Unlike HSP72, we detected expression of HSP47 in the basal epidermal layer as early as immediately posttreatment [Fig. 1(d)]. Although the level of epidermal expression did nost change through day 7 posttreatment, dermal expression progressively increased in a fashion similar to that observed for HSP72 (Figs. 1-4). Dermal expression of both HSPs was mainly surrounding the CDZ. Our results are in contrast to those of Laubach et al., who could only detect expression at day 1 posttreatment. ${ }^{16}$ The reason for this discrepancy may be due to the lower $(5 \mathrm{~mJ})$ energy treatment tested in their study. Thus, we are the first to demonstrate 


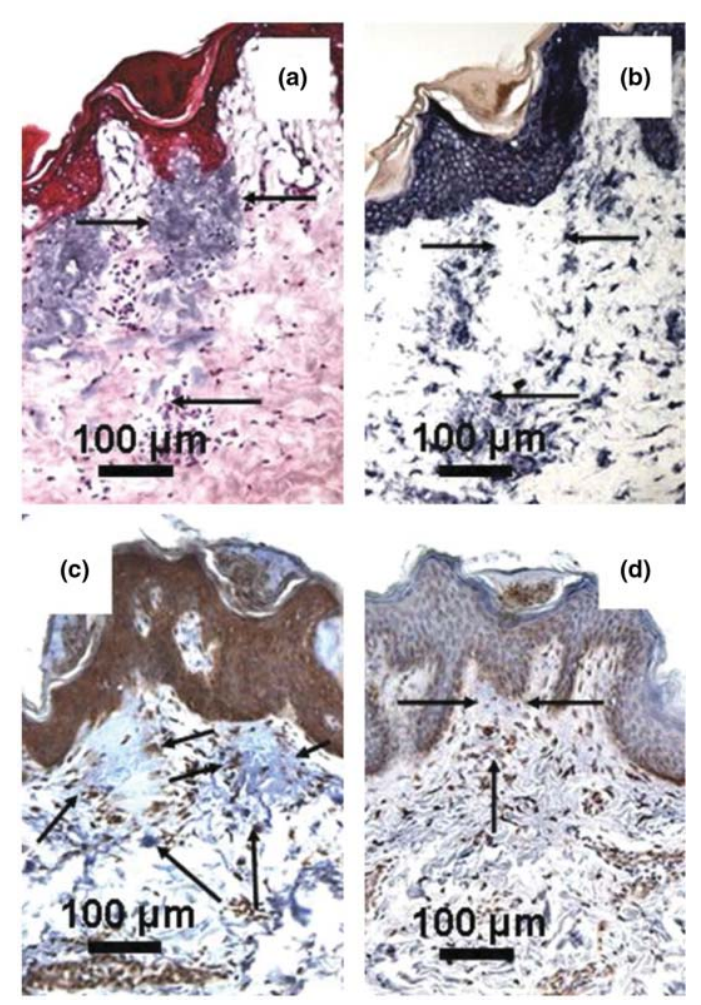

Fig. 4 Immunohistochemistry of human skin 7 days post-NFR treatment. In vivo human skin was treated with a 1550-nm infrared fractionated laser then biopsied immediately posttreatment. Tissue was stained with (a) H\&E, (b) LDH, (c) anti-HSP72 antibody, or (d) anti-HSP47 antibody. Brown staining in panels (c) and (d) represents positive immunoreactivity. Original magnification x 10 .

persistent expression of HSP72 and 47 beyond day 1 post-NFR treatment.

FP enables laser surgeons to achieve higher energy treatments through its ability to deliver electromagnetic energy to a fraction of the overall target tissue. The zones of sparing are critical to rapid wound healing and more robust dermal remodeling response. Histological data obtained in the current study showed the ZCD exceeded the CDZ by 19 to $55 \%$ immediately posttreatment, with the two zones converging by day 7 posttreatment (Fig. 5 and Table 2). Thus, it appears that the margins of the denatured zone become populated with viable dermal fibroblasts within 1 week of treatment. The origin of these cells is likely to be progenitor stem cells that homed to the site of injury from the systemic circulation, and then underwent differentiation into dermal fibroblasts.

Consistent with our previous findings, ${ }^{13}$ we also observed subepidermal clefting to be more prominent in paraffin sectioned slides [Figs. 1(a), 1(c), and 1(d)] than in their frozen section counterparts [Fig. 1(b)]. This difference was more obvious by day 1 posttreatment, at which time the epidermis had regained basophilic staining and the DE junction could be more clearly visualized [Fig. 2(b) compared to Figs. 2(a), 2(c), and 2(d)]. Our data suggested that both processing artifacts as well as laser-induced weakening contribute to the observed subepidermal clefting. This is in contrast to Laubach et al., ${ }^{16}$ who concluded that clefting was entirely due to thermal injury. One difference between our studies is the fact that we spanned pulse

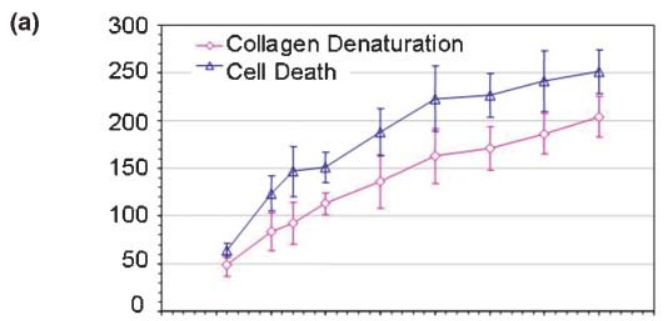

(b)

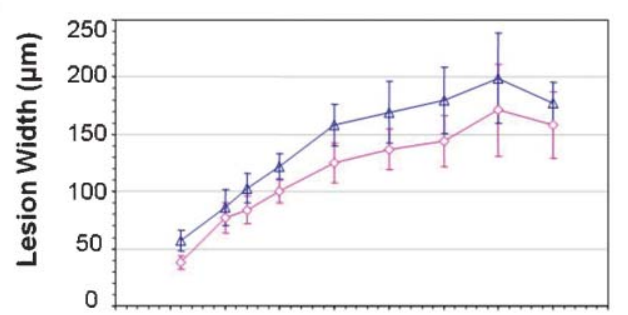

(c)

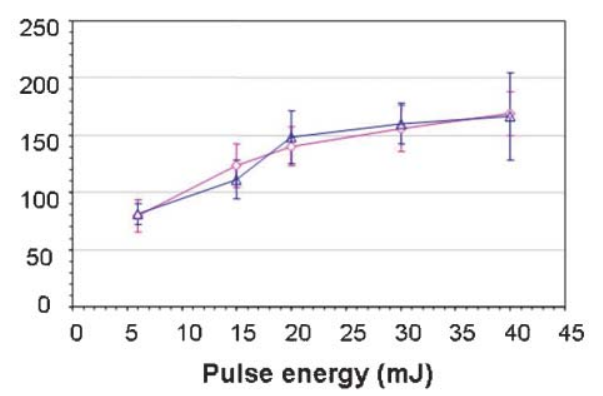

Fig. 5 Plots of mean MTZ width (a) immediately, 1 day, (b) or (c) 7 days following in vivo treatment of human skin with NFR treatment at varying pulse energies. Results represent the means \pm SE (Standard error) of $50 \mathrm{CDZ}$ and ZCD measurements obtained from H\&E \& LDH stained sections, respectively.

energies from 6 to $40 \mathrm{~mJ}$, whereas they only used the 5-mJ setting. Although higher energy settings may lead to increased thermal injury and thus separation of the DE junction, they also may induce a more robust epidermal repair process. Indeed, we found that a threshold pulse energy (data not shown) exists beyond which injury exceeds reparative capacity. For the range of pulse energies tested, we found that the DE junction was almost fully restored by day 3 and completely repaired by day 7 posttreatment (Fig. 3). These findings are in line with Laubach et al., who reported the presence of subepidermal clefting through day 5 posttreatment. ${ }^{16}$

In conclusion, our data suggest a temporal heat shock response that begins with induction of HSP72 expression approximately $24 \mathrm{~h}$ post-NFR treatment, followed by HSP47 within $48 \mathrm{~h}$. Unexpectedly, we observed persistent upregulation of both HSP72 and 47 at 7 days posttreatment, suggesting a continued role in the wound healing process beyond the traditionally accepted acute phase. The expression pattern of HSPs beyond 7 days posttreatment deserves further investigation, and is the subject of our ongoing studies as these data will provide further insight regarding their role in the long-term wound healing process post-NFR treatment. Notwithstanding, we also demonstrated that convergence of H\&E and LDH lesion dimensions occurred by 7 days post-NFR, as the wound healing response replenished the region surrounding MTZs with viable cells. Thus, NFR skin treatment induces a vigorous HSP-dependent epidermal and dermal remodeling process that restores 
cellular viability by day 7 posttreatment. In conclusion, our results suggest that (1) HSPs may represent more appropriate biomarkers for the treatment parameter optimization process than do traditional markers such as denatured collagen or cell death, (2) longer time intervals between consecutive NFR treatments should be considered, and (3) HSPs may better predict the therapeutic index of the device, and therefore should be used during device design to determine the choice of preselected treatment settings. This will enable laser surgeons to more safely deliver the desired levels of clinical efficacy to their patients.

\section{Acknowledgments}

We thank R. Kehl Sink, PhD, Diana Lac, BS, and Bhumika Kapadia, BS (all of Solta Medical, Inc., Hayward, California) for their technical assistance and intellectual contributions.

\section{References}

1. R. R. Anderson and J. A. Parrish, "Selective photothermolysis: precise microsurgery, by selection absorption of pulsed irradiation," Science 220, 524-527 (1983).

2. R. A. Weiss, M. A. Weiss, K. L. Beasley, and G. Munavalli, "Our approach to non-ablative treatment of photoaging," Laser Surg. Med. 37, 2-8 (2005).

3. D. Manstein, G. C. Herron, R. K. Sink, H. Tanner, and R. R. Anderson, "Fractional photothermolysis: a new concept for cutaneous remodeling using microscopic patterns of thermal injury," Lasers Surg. Med. 34, 426-438 (2004).

4. B. M. Hantash and M. B. Mahmood, "Fractional photothermolysis: a novel aesthetic laser surgery modality," Dermatol. Surg. 33, 525-534 (2007).

5. K. H. Kim, G. H. Fisher, L. J. Bernstein, S. Bangesh, G. Skover, and R. G. Geronemus, "Treatment of acneiform scars with fractional photothermolysis," Lasers Surg. Med. S17, 93 (2005).

6. D. S. Behroozan, L. H. Goldberg, T. Dai, R. G. Geronemus, and P. M. Friedman, "Fractional photothermolysis for the treatment of surgical scars: a case report," J. Cosmet. Laser. Ther. 8, 35-38 (2006).

7. T. Hasegawa, T. Matsukura, Y. Mizuno, Y. Suga, H. Ogawa, and S. Ikeda, "Clinical trial of a laser device called fractional photothermolysis system for acne scars," Dermatology 33, 623-627 (2006).

8. A. S. Glaich, Z. Rahman, L. H. Goldberg, and P. M. Friedman, "Fractional resurfacing for the treatment of hypopigmented scars: a pilot study," Dermatol. Surg. 33, 289-294 (2007).
9. A. Capon, E. Souil, B. Gauthier, C. Sumian, M. Bachelet, B. Buys, B. S. Polla, and S. Mordon, "Laser assisted skin closure (LASC) by using a 815 -nm diode-laser system accelerates and improves wound healing," Lasers Surg. Med. 28, 168-75 (2001).

10. S. D. DeCoste, W. Farinelli, T. Flotte, and R. R. Anderson, "Dyeenhanced laser welding for skin closure," Lasers Surg. Med. 12, 25-32 (1992).

11. D. P. Poppas, J. M. Massicotte, R. B. Stewart, A. B. Roberts, A. Atala, A. B. Retik, and M. R. Freeman, "Human albumin solder supplemented with TGF-beta 1 accelerates healing following laser welded wound closure," Lasers Surg. Med. 19, 360-368 (1996).

12. T. M. Wider, S. K. Libutti, D. P. Greenwald, M. C. Oz, J. S. Yager, M. R. Treat, and N. E. Hugo, "Skin closure with dye-enhanced laser welding and fibrinogen," Plast. Reconstr. Surg. 88, 1018-1025 (1991).

13. V. P. Bedi, R. K. Sink, K. F. Chan, B. M. Hantash, G. S. Herron, Z. Rahman, S. K. Struck, and C. B. Zachary, "The effects of pulse energy variations on the dimensions of microscopic thermal treatment zones in nonablative fractional resurfacing," Lasers Surg. Med. 39, 144-155 (2007).

14. J. G. Kiang and G. C. Tsokos, "Heat shock protein $70 \mathrm{kDa}$ : molecular biology, biochemistry, and physiology," Pharmacol. Ther. 80, 183-201 (1998).

15. A. F. Laplante, V. Moulin, F. A. Auger, J. Landry, H. Li, G. Morrow, R. M. Tanguay, and L. Germain, "Expression of heat shock proteins in mouse skin during wound healing," J. Histochem. Cytochem. 46, 1291-1301 (1998).

16. H. J. Laubach, Z. Tannous, R. R. Anderson, and D. Manstein, "Skin responses to fractional photothermolysis" Lasers Surg. Med. 38, 142-9 (2006).

17. S. A. Brown, J. P. Farkas, C. Arnold, D. A. Hatef, J. Kim, J. Hoopman, and J. M. Kenkel, "Heat shock proteins 47 and 70 expression in rodent skin model as a function of contact cooling temperature: are we overcooling our target," Lasers Surg. Med. 39, 504-512 (2007).

18. B. M. Hantash, V. P. Bedi, K. F. Chan, and C. B. Zachary, "Ex vivo histological characterization of a novel ablative fractional resurfacing device," Lasers Surg. Med. 39, 87-95 (2007).

19. B. M. Hantash, V. P. Bedi, B. Kapadia, Z. Rahman, K. Jiang, H. Tanner, K. F. Chan, and C. B. Zachary, "In vivo histological evaluation of a novel ablative fractional resurfacing device," Lasers Surg. Med. 39, 96-107 (2007).

20. H. Takechi, K. Hirayoshi, A. Nakai, H. Kudo, S. Saga, and K. Nagata, "Molecular cloning of a mouse $47-\mathrm{kDa}$ heat-shock protein HSP47, a collagen-binding stress protein, and its expression during the differentiation of F9 teratocarcinoma cells," Eur. J. Biochem. 206, 323-329 (1992). 